\title{
乙型肝炎病毒X蛋白调节TRAIL诱导的 肝细胞调亡*
}

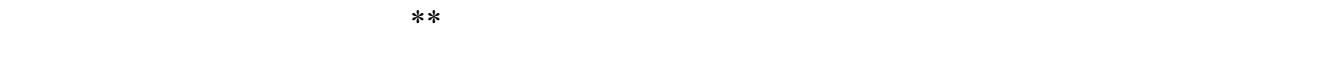

(1) 山东大学医学院免疫学研究所, 济南 250012; (2) Department of Pathology and Laboratory Medicine, University of Pennsylvania, PA19104, USA)

摘要 观察乙型肝炎病毒 $\mathrm{X}$ 蛋白 $(\mathrm{HBx})$ 对肿瘤坏死因子相关的调亡诱导配体(TNF-related apoptosis-inducing ligand, TRAIL)诱导肝细胞调亡的影响并初步探讨其分子机制. 构建包含 HBx 基因的真核表达载体 pcDNA-HBx, 转染 BEL7402 肝癌细胞, 建立可稳定表达 HBx 的肝癌细胞系 BEL7402-HBx, 同时设立空载体 pcDNA3 转染对照组细胞 BEL7402-cDNA3. 台盼蓝染色计数, Caspase3 活性检测和 TUNEL 法检测 TRAIL 诱导 BEL7402, BEL7402-cDNA3, BEL7402-HBx 细 胞凋亡的情况, 并通过流式细胞术分析 3 组细胞表面 TRAIL 受体的表达水平. 此外, 利用硫代反 义寡核苷酸封闭 HBV 全基因转染肝癌细胞系 HepG2.2.15 中 HBx 蛋白的表达, 观察阻断前后对 TRAIL 诱导调亡敏感性的改变, 进一步反向验证 HBx 对 TRAIL 诱导调亡的调节作用. 台盼蓝染 色计数提示 TRAIL对 BEL7402, BEL7402-cDNA3, BEL7402-HBx 均有剂量依赖性的细胞毒作 用, 但在相同浓度 TRAIL 作用下, BEL7402-HBx 细胞较 BEL7402, BEL7402-cDNA3 细胞有更 高的敏感性. Caspase3 活性检测结果分析发现, TRAIL 作用后 BEL7402-HBx 细胞在较短时间内 有更高的 Caspase3 活化水平. TUNEL 结果显示, $10 \mu \mathrm{g} / \mathrm{LTRAIL}$ 作用下, BEL7402-HBx 细胞凋亡率 可达 $(41.4 \pm 7.2 \%$, 显著高于对照组细胞. 反义封闭 HepG2.2.15 细胞中 $\mathrm{HBx}$ 基因的表达可部分阻 断 TRAIL 诱导的调亡. 两组实验结果均显示 HBx 的表达变化并不影响细胞表面 TRAIL 受体的表 达模式. HBx 蛋白参与调节 TRAIL 诱导的细胞调亡, 可能在 HBV 相关疾病的发生中起一定作用, 这一作用与 TRAIL 受体表达水平无关. 从两个不同的侧面证实了 HBx 对 TRAIL 诱导细胞调亡的 调节作用, 为进一步论证调亡失衡在 HBV 感染相关肝炎及肝癌发生中的作用提供了新的 论据. 


\section{关键词 凋亡 TRAIL HBx 蛋白 硫代反义寡核苷酸}

肿瘤坏死因子相关的调亡诱导配体(TNF-related apoptosis-inducing ligand, TRAIL)属肿瘤坏死因子超 家族成员, 可特异性诱导肿瘤细胞或病毒感染的细 胞调亡而正常组织细胞不受影响 ${ }^{[1,2]}$, 目前已成为肿 瘤生物治疗的候选分子之一。

众多因素参与调节细胞对 TRAIL 诱导调亡的敏 感性, 包括细胞因子、病毒感染、药物、射线等. 研 究表明, 某些病毒或病毒基因编码的蛋白可影响 TRAIL 诱导的细胞调亡. 呼吸道合胞病毒、人类免疫 缺陷病毒、呼肠弧病毒可上调宿主细胞对 TRAIL 诱 导调亡的敏感性，而人乳头瘤病毒编码的 E5 蛋白及 腺病毒蛋白则抑制 TRAIL 诱导的宿主细胞的调亡. HBV 感染对 TRAIL 诱导细胞调亡的影响目前尚存在 争议. HBx 是由 HBV 基因组编码的病毒蛋白之一, 在病毒复制和感染肝细胞癌变中起非常重要的作用. 研究表明, HBx 通过激活细胞内癌基因的表达及信 号传导途径, 导致细胞增殖甚至癌变 ${ }^{[3,4]}$. 然而, 亦有 研究发现, HBx蛋白尚可诱导细胞调亡 ${ }^{[5]}$ 或提高细胞 对某些调亡刺激信号(如TNF)的敏感性 ${ }^{[6]}$.

本研究旨在探讨 HBx 是否参与调节 TRAIL 诱导 的细胞调亡反应. 文中同时采用了两种研究方案: 即 在肝癌细胞中过表达 HBx 蛋白和利用反义寡核苷酸 封闭 HBV 全基因转染细胞中 $\mathrm{HBx}$ 的表达，从两个不 同的角度更加准确地说明 HBx 在 TRAIL 诱导调亡中 的作用.

\section{1 材料和方法}

\subsection{HBX 基因的表达对 TRAIL 诱导 BEL7402 细 胞调亡的影响}

(1) 细胞和细胞培养: 肝癌细胞系BEL7402 为本 室保存细胞株，传代于含 $10 \%$ 胎牛血清的 RPMI 1640 (购自 GIBCO公司)完全培养基中, 在 $37^{\circ} \mathrm{C}, 5 \%$ $\mathrm{CO}_{2}$ 细胞培养箱内培养. BEL7402 在培养瓶内呈单层 贴壁生长，约每 4 天经 $0.25 \%$ 胰酶加入 $0.02 \%$ EDTA消 化传代 1 次.

(2) PCR 扩增 HBx 基因片段: 质粒 pUC19/3HBV 包含 3 个拷贝的 $\mathrm{adr}$ 亚型 $\mathrm{HBV}$ 全基因序列, 由日本
Oita Medical 大学 Akira Nishizono 教授馈赠. 以 $\mathrm{pUC} 19 / 3 \mathrm{HBV}$ 为模板，设计合成针对 $\mathrm{HBx}$ 基因片段 的 PCR 引物序列: 上游引物(包含 HindIII酶切位点): $5^{\prime}$-gtaagcttatggctgctagggtgt-3', 下游引物(包含 Xba I 酶切位点): 5'-ctctagactaggcagaggtgaaaaag-3'. PCR 在 $25 \mu \mathrm{L}$ 的反应体系中进行: 模板 $1 \mu \mathrm{L}, \mathrm{PCR} 反$ 应缓冲液 $2.5 \mu \mathrm{L}, \mathrm{MgCl}_{2} 1.5 \mu \mathrm{L}, \mathrm{dNTP} 0.5 \mu \mathrm{L}$, 引物各 $0.5 \mu \mathrm{L}$, 耐热DNA聚合酶 $0.5 \mu \mathrm{L}$. PCR反应条件如下: $95^{\circ} \mathrm{C}$ 预 变性 $3 \mathrm{~min} ; 95^{\circ} \mathrm{C}$ 变性 $45 \mathrm{~s}, 62^{\circ} \mathrm{C}$ 退火 $1 \mathrm{~min}, 72^{\circ} \mathrm{C}$ 延伸 $45 \mathrm{~s}$, 共循环 40 次; 最后 $72^{\circ} \mathrm{C}$ 延伸 $5 \mathrm{~min}$.

(3) HBx 表达载体的构建和鉴定：上述 PCR 反应 产物经 HindIII和 Xba I 双酶切, 酶切片段定向克隆入 经同样酶切的真核表达载体 pcDNA3 (购自 Invitrogen 公司)内, 阳性重组子经酶切、DNA 测序鉴定获得, 并被命名为 pcDNA-HBx.

(4) 脂质体介导的稳定转染: pcDNA3 空载体或 pcDNA-HBx重组子稳定转染入BEL7402 细胞. 脂质 体(Lipofectamine ${ }^{\mathrm{TM}} 2000$ 购自 GIBCO公司)介导的基 因转染按照试剂盒说明书进行。转染前一天将 BEL7402 细胞以每孔 $1.5 \times 10^{5}$ 的浓度接种于 24 孔板内, 培养 $20 \mathrm{~h}$. 待细胞生长至 $85 \%$ 汇片时, 移去原有培养 液, 加入转染混合液(即包含有 $1 \mu \mathrm{g}$ 目的 DNA, $2 \mu \mathrm{L}$ 脂质体的无血清 Opti-MEM 培养基), $37^{\circ} \mathrm{C}$ 培养 6 h. 更换 $0.5 \mathrm{~mL}$ 完全培养基, 继续培养 $24 \mathrm{~h}$. 稳定转 染的阳性克隆经包含 $380 \mu \mathrm{g} / \mathrm{mLG} 418$ (购自 GIBCO 公 司)的完全培养基笁选 4 周获得, 分别命名为 BEL 7402cDNA3, BEL7402-HBx.

(5) 逆转录 PCR 鉴定稳定转染细胞株: 收集 BEL7402, BEL7402-cDNA3, BEL7402-HBx 细胞, 利 用 Trizol 试剂(购自加拿大 Bio Basic 公司)抽提细胞总 RNA，具体步骤按照试剂盒说明书进行. RNA 浓度 经分光光度计(Eppendorf 公司)定量测定. 逆转录在 $20 \mu \mathrm{L}$ 反应体系中进行: 总 RNA1 $\mu \mathrm{g}$, 逆转录反应缓 冲液 $4 \mu \mathrm{L}$, dNTP $20 \mathrm{nmol}$, RNA 酶抑制剂 $20 \mathrm{U}, \mathrm{M}$ MLV 逆转录酶 $200 \mathrm{U}, 42^{\circ} \mathrm{C}$ 反应 $1 \mathrm{~h}$. 产物在 $70^{\circ} \mathrm{C}$ 变性 $10 \mathrm{~min}$ 后保存于 $4^{\circ} \mathrm{C}$. 以获得的 $\mathrm{cDNA}$ 为模板, $\mathrm{PCR}$ 扩增 $\beta$-actin 和 $\mathrm{HBx}$. HBx 的引物同前. 另设计合成针 
对管家基因 $\beta$-actin 的特异性引物 (上游引物：5'ACACTGTG CCCATCTACGAGGGG-3', 下游引物 : 5'- ATGATGGAGTTGAAGGTAGTTTCGTGG AT-3').

（6）TRAIL介导的细胞毒效应：BEL 7402, BEL7402-cDNA3, BEL7402-HBx细胞以每孔 $2 \times 10^{4}$ 个 细胞的浓度接种于 96 孔板, 培养 $20 \mathrm{~h}$ 后细胞生长至 对数期. 不同浓度 $(10,20,50,100,200,500 \mu \mathrm{g} / \mathrm{L})$ 的 TRAIL蛋白(购自Pepro Tech公司)分别加入至各组细 胞(同时设立不经TRAIL作用的对照组细胞), 继续培 养 $24 \mathrm{~h}$ 后, 台盼蓝染色计数法检测各孔细胞中的活细 胞数目. 具体步骤如下: 收集各孔细胞, 制备成 200 $\mu \mathrm{L}$ 的单细胞悬液, 并与等体积 $0.3 \%$ 的台盼蓝溶液(购 自Sigma公司)混合，显微镜下计数未经台盼蓝染色的 细胞数目. 计算细胞杀伤率 $(\mathrm{CR}=1-$ 实验组平均活细 胞数目 (对照组平均活细胞数目), 描绘生长曲线图. 结果为 3 次实验的平均值, 各组均设 3 个复孔. IC50 为细胞杀伤率为 $50 \%$ 时 T R A I L 蛋白的作用 浓度.

(7) TUNEL法检测细胞调亡: BEL7402, BEL7402cDNA3, BEL7402-HBx细胞以每孔 $2 \times 10^{5}$ 个细胞的浓 度接种于 24 孔板, 各孔加入 $10 \mu \mathrm{g} / \mathrm{L}$ TRAIL捊育 $24 \mathrm{~h}$. 收集细胞, TUNEL法检测细胞凋亡率(凋亡原位检测 试剂盒购自 Immunotech公司). 具体步骤如下: 细胞 以 $4 \%$ 多聚甲醛 $4{ }^{\circ} \mathrm{C}$ 固定 $30 \mathrm{~min}$, 用含 $0.2 \% \mathrm{BSA}$ 的 PBS洗涤两次; 再以 $70 \%$ 冷乙醇 $20^{\circ} \mathrm{C}$ 细胞膜打孔 30 $\min$, 用含 $0.2 \% \mathrm{BSA}$ 的PBS洗涤两次; 最后加入末端 脱氧核糖核苷酸转移酶反应剂(由末端脱氧核糖酸转 移酶, 酶缓冲液, FITC-dUTP以 18: 1: 1 的比例混合 而成) $37^{\circ} \mathrm{C}$ 孵育 $1 \mathrm{~h}$, 用含 $0.2 \% \mathrm{BSA}$ 的PBS洗涤两次, 细胞沉淀重悬于 $0.5 \mathrm{~mL}$ 含 $0.2 \% \mathrm{BSA}$ 的PBS溶液中, 上样流式细胞仪, 计数阳性细胞数目, 结果以阳性细 胞占总细胞的百分数表示细胞调亡率.

(8) Caspase 3 活性检测: BEL7402, BEL7402cDNA3, BEL7402-HBx细胞以每孔 $1 \times 10^{6}$ 个细胞的浓 度接种于 6 孔板, 各孔加入 $10 \mu \mathrm{g} / \mathrm{L}$ TRAIL分别孵育 $2,5,8,11,24 \mathrm{~h}$, 设阴性对照. 采用Caspase 3 活性检 测试剂盒(购自Clontech公司)测定Caspase3 的活化情 况. 具体方法如下：分别收集各组细胞, PBS洗涤 2
次, 加入细胞裂解缓冲液 $50 \mu \mathrm{L}$, 冰上捊育 $30 \mathrm{~min}$; $12000 \mathrm{r} / \mathrm{min}, 4^{\circ} \mathrm{C}$ 离心 $5 \mathrm{~min}$, 回收上清, 移至新的 Eppendorf管，每管依次加入 $50 \mu \mathrm{L}$ 的 $2 \times$ 反应缓冲液 (含 $10 \mathrm{mmol} / \mathrm{L}$ DTT), $1 \mathrm{mmoL} / \mathrm{L}$ Caspase-3 底物 DEVD-pNA $5 \mu \mathrm{L}, 37^{\circ} \mathrm{C}$ 睬育 $2 \mathrm{~h}$, 转移至 96 孔板中, 用酶标仪测定波长 $405 \mathrm{~nm}$ 的吸光度值 $\left(A_{405}\right)$, 表示 Caspase 3 的相对活性.

（9）流式细胞术检测TRAIL受体的表达：处于对 数生长期的BEL7402, BEL7402-cDNA3, BEL7402$\mathrm{HBx}$ 细胞胰酶消化制备单细胞悬液, 以冷PBS溶液洗 涤两次. $1 \times 10^{6}$ 细胞沉淀重悬于 $200 \mu \mathrm{L}$ PBS, 不同管 中分别加入小鼠抗DR4, DR5, DcR1, DcR2 的单克隆 抗体(购自第四军医大学, 以 1:200 的比例稀释), $4^{\circ} \mathrm{C}$ 孵育 $30 \mathrm{~min}$, 冷 PBS 洗涤两次. 细胞沉淀重悬于 含有 FITC 标记的兔抗鼠二抗的 PBS 溶液中, $4^{\circ} \mathrm{C}$ 捊育 $30 \mathrm{~min}$, 冷 PBS 洗涤两次, 最后将细胞沉淀溶于 $1 \mathrm{~mL}$ PBS 溶液中, 上样流式细胞仪, 检测样品平均荧光强 度.

\subsection{PS-asODNs/HBx 对 TRAIL 诱导 Hep G2.2.15 细胞调亡的影响}

（1）细胞和细胞培养: Hep G2.2.15 肝癌细胞株, 整合有 4 个拷贝的 HBV 基因组, 可完成病毒的复制 和抗原合成, 并产生完整的 Dane's 颗粒, 由北京医学 生物学研究所提供. 细胞传代培养于含有 $10 \%$ 胎牛 血清, $380 \mu \mathrm{g} / \mathrm{mL} \mathrm{G} 418$ 的 MEM 培养基中. MEM, 胎 牛血清和 G418 购自 GIBCO 公司.

（2）硫代寡核苷酸的合成: 设计互补于 $H B x$ 基因 (ayw1 亚型)翻译起始区的反义寡核苷酸序列 PSasODNs/HBx (5'-GCACA GCCTA GCAGC CAT-3'), 同 时设计 HBV 非互补随机序列 PS-rODNs (5'-TTGCC GAGCG GGGTA-3')作为对照. 以上鿒核苷酸序列由 上海博亚公司合成，并作硫代修饰。硫代寡核苷酸粉 末溶于 MEM 完全培养基中, 终浓度为 $10 \mu \mathrm{mol} / \mathrm{L}$.

(3) PS-asODNs/HBx体外抗病毒试验: Hep G2.2.15 细胞以每孔 $2 \times 10^{4}$ 个细胞的浓度接种 96 孔板, 常规培养 $20 \mathrm{~h}$ 后, 将PS-asODNs/HBx 或 PS-rODNs 按 $10 \mu \mathrm{mol} / \mathrm{L}$ 加入各孔, 继续孵育 $24 \mathrm{~h}$ 或 $48 \mathrm{~h}$. 收集培 养液上清 $200 \mu \mathrm{L}$, 用ELISA试剂盒(深圳丽珠集团) 
检测 HBsAg 和 HBeAg 的表达情况，酶标仪测定 450 $\mathrm{nm}$ 波长下的吸光值. 抑制率 $(\mathrm{IR})=(1-$ 实验组平均吸 光值/对照组平均吸光值) $\times 100 \%$. HBV DNA 的产量由 苂光定量 PCR 检测试剂盒测得, 结果以 copies $/ \mathrm{mL}$ 表 示. 抑制率 $(\mathrm{SR})=(1-$ 实验组平均拷贝数/对照组平均 拷贝数) $\times 100 \%$. 结果为 3 次实验的平均值, 各组均 设 3 个复孔.

(4) PS-asODNs/HBx对Hep G2.2.15 细胞调亡的 影响: Hep G2.2.15 细胞以每孔 $2 \times 10^{5}$ 个细胞的浓度接 种 24 孔板, PS-asODNs/HBx或 PS-rODNs $(10 \mu \mathrm{mol} / \mathrm{L})$ 作用 $24 \mathrm{~h}$ 后, 各孔加入 $100 \mu \mathrm{g} / \mathrm{L}$ TRAIL继续孵育 $24 \mathrm{~h}$. 收集细胞, TUNEL法检测细胞凋亡率(方法见第 1.1 节 (6)).

(5) PS-asODNs/HBx 对 TRAIL 受体表达的影响: Hep G2.2.15 细胞经 PS-asODNs/HBx 或 PS-rODNs 作 用 $24 \mathrm{~h}$ 后, 胰酶消化制备单细胞悬液, 以冷 PBS 溶液 洗涤两次, 流式细胞术检测 TRAIL 受体的表达情况 (方法见第 1.1 小节(9)).

\section{3 统计学分析}

应用统计学软件SPSS10.0 对各实验结果进行统 计学分析. 细胞杀伤率和细胞调亡率经 $\chi^{2}$ 检验比较, $P<0.05$ 被定为有统计学意义.

\section{2 结果}

\subsection{HBx 可增强 TRAIL 诱导的 BEL7402 细胞的 调亡}

(1) HBx 表达载体的构建: PCR 成功扩增获得 $\mathrm{HBx}$ 基因片段, $2 \%$ 琼脂糖凝胶电泳确定 PCR 产物分 子量大小为 $515 \mathrm{bp}$ 左右(图 1). 产物经酶切定向克隆 入 pcDNA3 内. 阳性重组子经 HindIII和 Xba I 双酶切 鉴定分子量大小(图 2), 并经 DNA 测序确定插入片段 的正确性.

(2) 建立 BEL7402-cDNA3, BEL7402-HBx 细胞 株: pcDNA3 或 pcDNA-HBx 转染的 BEL7402 细胞经 含有 G418 的完全培养基篎选 4 周获得阳性细胞克隆, 稳定传代建立 BEL7402-cDNA3, BEL7402-HBx 细胞 株.

(3) RT-PCR 检测 HBx mRNA 的表达: 各组细胞

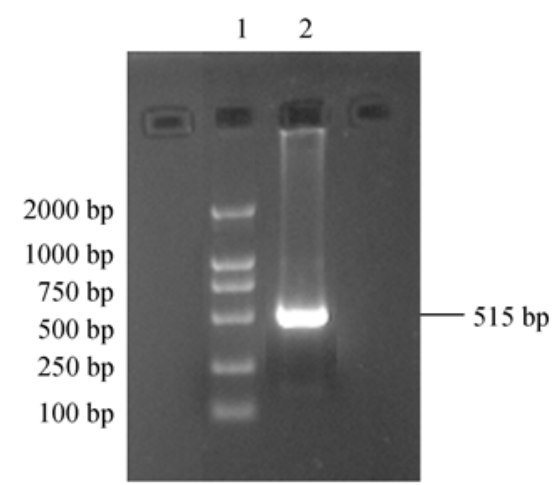

图 1 PCR 扩增 $\mathrm{HBx}$ 基因片段 1 示 Marker 2000; 2 示 $\mathrm{HBx}$ 基因

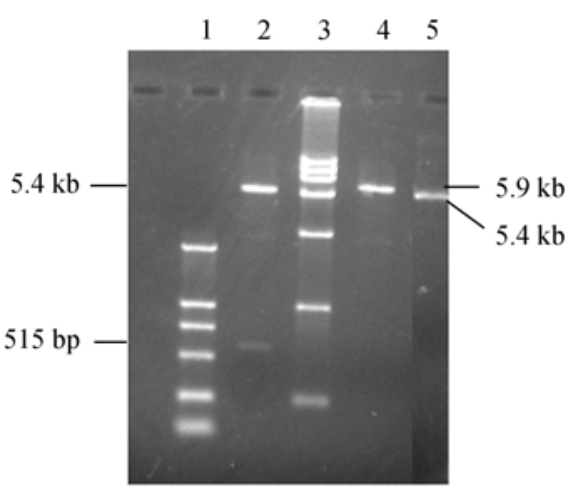

图 2 酶切鉴定 pcDNA-HBx 重组子 1 示 Marker 2000; 2 示 pcDNA-HBx 经 HindIII+Xba I 双酶切; 3 示 Marker 15000; 4 示 pcDNA-HBx 经 HindIII单酶切; 5 示 pcDNA3 经 HindIII单酶切

RNA经分光光度计分析, $A_{260} / A_{280}$ 的比值在 1.8 2.0 范 围内. 以上述总 RNA为模板逆转录获得 $\mathrm{cDNA}$, 并经 $\mathrm{PCR}$ 扩增 $\beta$-actin基因. 琼脂糖凝胶电泳可见分子量大 小为 $367 \mathrm{bp}$ 的条带(图 3). 检测 3 组细胞中 $\mathrm{HBx}$

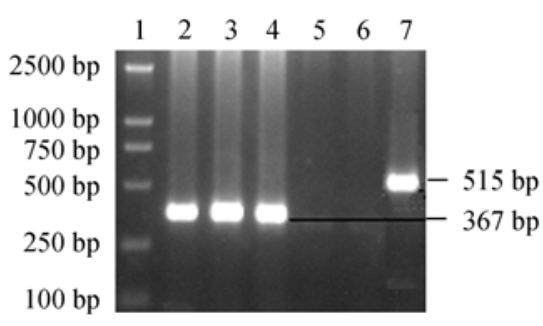

图 3 RT-PCR 检测 HBx 的表达

1 示 Marker 2000; 2 4 示由 BEL7402, BEL7402-cDNA3, BEL7402HBx mRNA 扩增的 $\beta$-actin 片段; 5 7 示由 BEL7402, BEL7402-cDNA3, BEL7402-HBx mRNA 扩增的 HBx 片段 
mRNA 的表达, 结果显示仅 BEL7402-HBx 细胞有特 异性的表达，而在对照组细胞 BEL7402，BEL7402cDNA3 中未见表达.

（4） TRAIL 剂量依赖性细胞毒效应: 台盼蓝染色 计数结果显示, BEL7402 及其稳定转染细胞株均对 TRAIL 有较高的敏感性, 且随着 TRAIL 作用浓度的 增加其细胞毒效应呈现明显的剂量依赖性(图 4). 在 相同作用浓度下, TRAIL 蛋白对 BEL7402-HBx 细胞 较 BEL7402, BEL7402-cDNA3 有更高的杀伤率, IC50 分别为 17 和 $35 \mu \mathrm{g} / \mathrm{L}$.

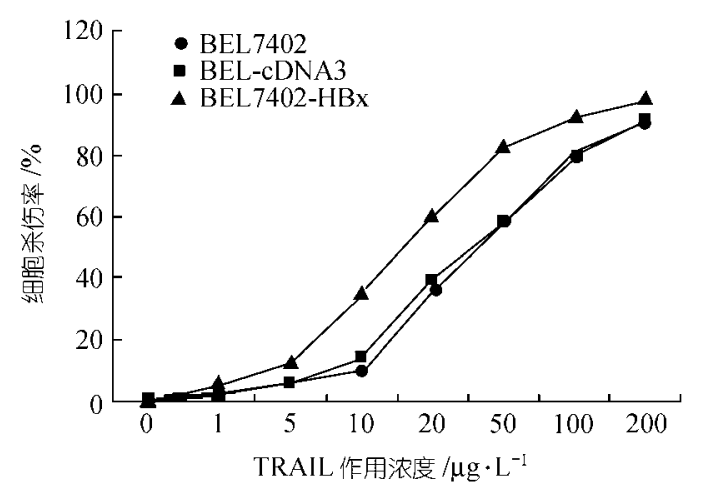

图 4 TRAIL 的细胞毒效应

(5) HBx 可提高 TRAIL 诱导的 BEL7402 细胞的 调亡率: $10 \mu \mathrm{g} / \mathrm{L}$ TRAIL 作用于 BEL7402, BEL7402cDNA3, BEL7402-HBx 细胞, $24 \mathrm{~h}$ 后 TUNEL 法检测 细胞调亡率，结果显示, BEL7402, BEL7402-cDNA3 的细胞调亡率分别为 $(13.8 \% \pm 6.5) \%$ 和 $(14.2 \pm 4.7) \%$, 显著低于 $\operatorname{BEL} 7402-\mathrm{HBx}(41.4 \% \pm 7.2) \%(P<0.001)$, 而 BEL7402, BEL7402-cDNA3 两组细胞间调亡率无显 著差异(图 5). 也就是说, HBx 可在一定程度上提高 BEL7402 细胞对 TRAIL 诱导调亡的敏感性.

(6) HBx 对 Caspase 3 活化的影响: Caspase 3 活性 检测结果显示, TRAIL 作用 BEL7402, BEL7402cDNA3, BEL7402-HBx 细胞后, 均可在一定程度上活
化 Caspase3, 且在作用 $8 \mathrm{~h}$ 左右达到最高峰, 但 BEL7402HBx 细胞 Caspase3 的活化水平显著高于 BEL7402, BEL7402-cDNA3 对照组细胞 $(P<0.05)$ (图 6).

(7) TRAIL 受体的表达变化: 流式细胞术检测 BEL7402, BEL7402-cDNA3, BEL7402-HBx 细胞表面 TRAIL 受体蛋白质水平的表达情况, 结果显示, 3 组 细胞间各受体表达均无明显差异(图 7).

\subsection{PS-asODN/HBx 可部分抑制 TRAIL 诱导的 Hep G2.2.15 细胞的凋亡}

(1) PS-asODNs/HBx 可抑制 HBV 抗原的合成及 病毒 DNA 的复制: 如表 1 所示, PS-asODNs/HBx 可 显著抑制 HBsAg, HBeAg 的合成和病毒 DNA 的复制. 作用 $24 \mathrm{~h}$ 后，对 HBsAg, HBeAg 合成的抑制率分别 为 $(28.1 \% \pm 4.2) \%,(41.2 \% \pm 5.7) \%$; 作用 $48 \mathrm{~h}$ 后, 对 $\mathrm{HBsAg}, \mathrm{HBeAg}$ 合成的抑制率分别为 $(40.3 \% \pm 3.2) \%$, $(38.1 \% \pm 5.4) \%$ ，显著高于 PS-rODNs 作用组细胞. 分 析表明二者差异有统计学意义 $(P<0.01)$. 荧光定量 PCR 结果显示与 PS-rODNs 作用组相比, PS-asODNs/ $\mathrm{HBx}$ 尚可显著降低 Hep G2.2.15 细胞培养上清中 HBV DNA 的含量(表 2), 作用 $24,48 \mathrm{~h}$ 后的抑制率分别为 $30.2 \%, 77.1 \%$.

(2) PS-asODNs/HBx 可逆转 Hep G2.2.15 细胞对 TRAIL 诱导调亡的敏感性: Hep G2.2.15 细胞先经 PS-asODNs/HBx 或 PS-rODNs 作用 $24 \mathrm{~h}$ 后, 分别加入 $100 \mu \mathrm{g} / \mathrm{L}$ TRAIL 继续作用 $24 \mathrm{~h}$, TUNEL 结果显示, PS-asODNs/HBx 与 TRAIL 联合作用组调亡率为 $(18.5 \% \pm 9.5) \%$ ，显著低于 PS-rODNs 与 TRAIL 联合作 用组 $(62.1 \% \pm 12) \%$ (图 8), 结果表明, PS-asODNs/HBx 可部分逆转 Hep G2.2.15 细胞对 TRAIL 诱导凋亡的敏 感性, 逆转率可达 $70.2 \%(P<0.001)$.

(3) PS-asODNs/HBx 对细胞表面 TRAIL 受体的表 达无显著影响: 经 PS-asODNs/HBx 或 PS-rODNs 作用

表 1 PS-asODNs/HBx 对HBV抗原合成的影响 ${ }^{a)}$

\begin{tabular}{|c|c|c|c|c|}
\hline \multirow{2}{*}{ PS-ODNs } & \multicolumn{2}{|c|}{$24 \mathrm{~h}$ 的抑制率/\% } & \multicolumn{2}{|c|}{$48 \mathrm{~h}$ 的抑制率/\% } \\
\hline & HBsAg & HBeAg & HBsAg & HBeAg \\
\hline PS-asODNs/HBx & $28.1 \pm 4.2 *$ & $41.2 \pm 5.7^{*}$ & $40.3 \pm 3.2 *$ & $38.1 \pm 5.4^{*}$ \\
\hline PS-rODNs & $7.2 \pm 1.5$ & $8.1 \pm 2.1$ & $5 \pm 1.4$ & $6.4 \pm 2.3$ \\
\hline
\end{tabular}

a) 结果以均数 \pm 标准差表示. *示与 PS-rODNs作用组相比, $P<0.01$ 


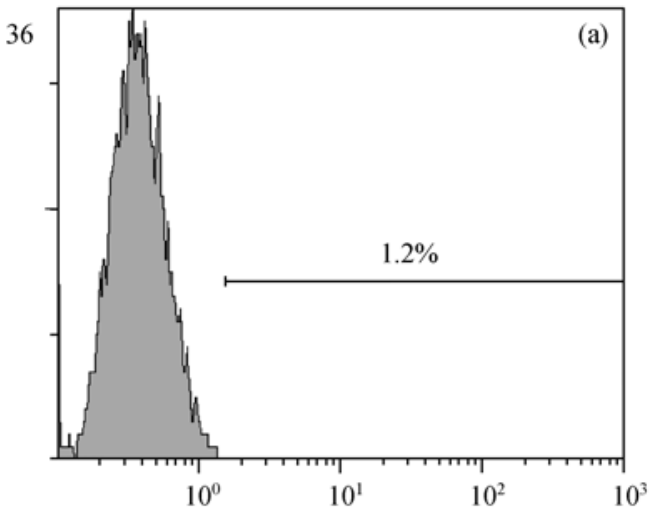

61

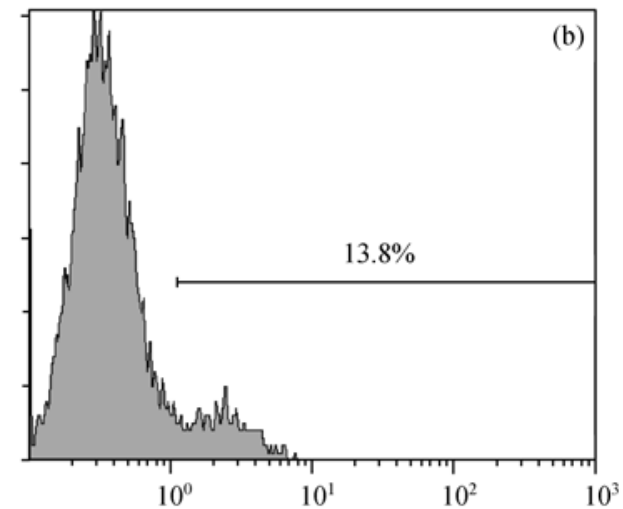

69

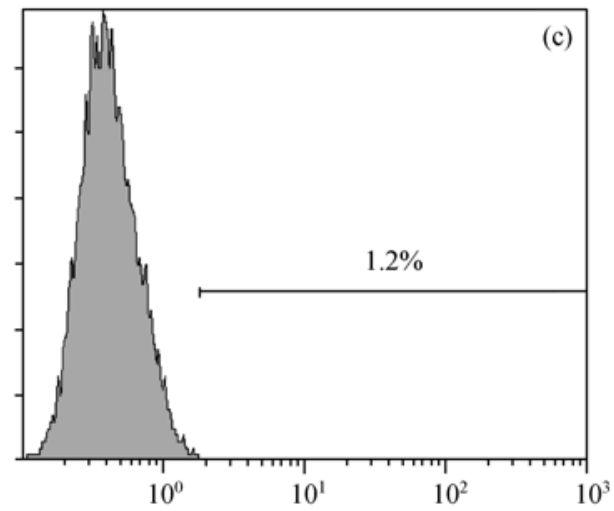

73

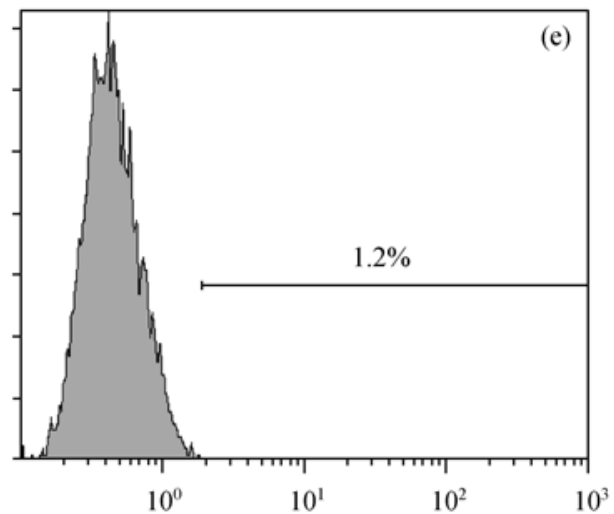

77

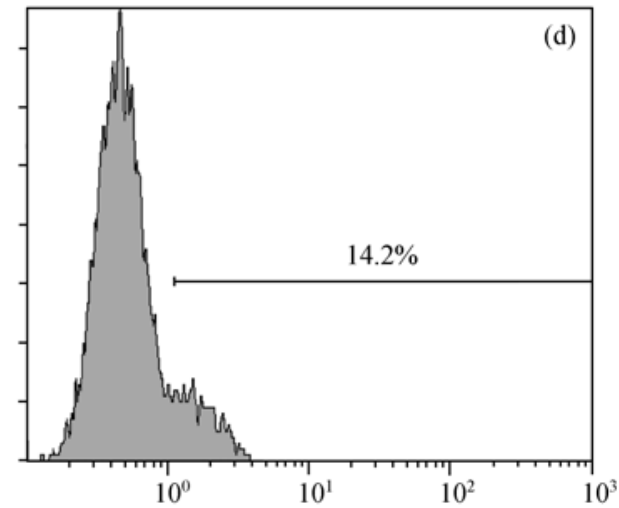

50

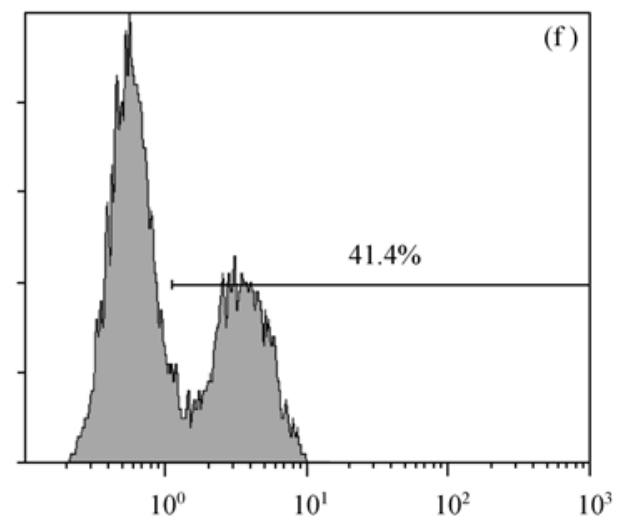

图 5 TUNEL 法检测细胞调亡

(a) BEL7402 对照组; (b) TRAIL(10 $\mu \mathrm{g} / \mathrm{L}$ )作用的 BEL7402 细胞; (c) BEL 7402-cDNA3 对照组; (d) TRAIL(10 $\mu \mathrm{g} / \mathrm{L})$ 作用的BEL 7402- cDNA3 细胞; (e) BEL 7402-HBx 对照组; (f) TRAIL $(10 \mu \mathrm{g} / \mathrm{L}$ ) 作用的 BEL 7402-HBx 细胞; (d) 组与 (b) 组相比, $P>0.05$; (f) 组与 (b)组和(c)组相比, $P<0.01$

$24 \mathrm{~h}$ 后, 流式细胞术检测 Hep G2.2.15 细胞表面 TRAIL 受体的表达水平, 结果显示, PS-asODNs/HBx 作用组 各受体表达水平与对照组相比无显著差异(图 9).

\section{3 讨论}

众多研究表明, HBV 感染所致肝细胞损伤与其 所诱导的肝细胞调亡密切相关. FasL/Fas, TNF $\alpha / T N F-$ 


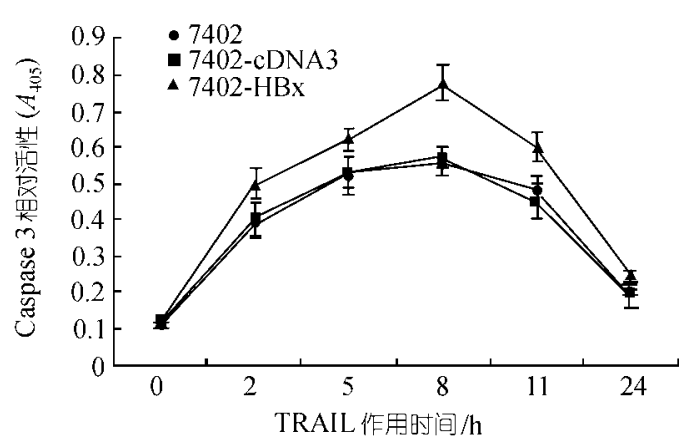

图 6 Caspase 3 活性检测

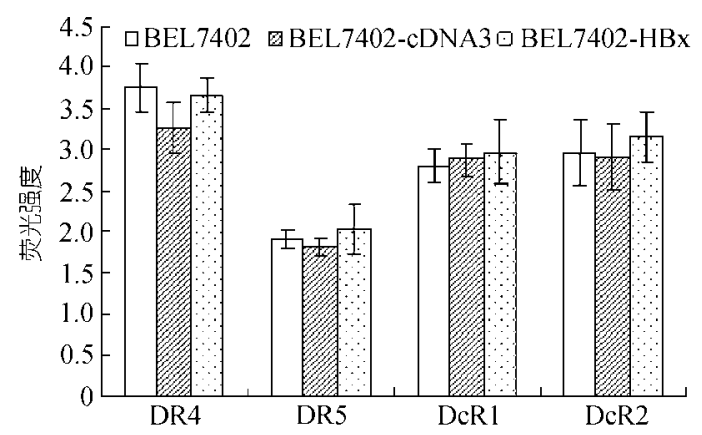

图 7 BEL7402 及其稳定转染细胞 TRAIL 受体的表达

R1 已被证实在该过程中发挥重要作用 ${ }^{[7,8]}$. 已有研究 表明, HBV感染可影响TRAIL诱导的细胞调亡, 但其 详尽的作用目前尚不十分明确. HBV基因组可编码多 种病毒蛋白, 其中HBx蛋白序列在哺乳动物嗜肝病毒 科高度保守，且被证实在 HBV复制和感染肝细胞癌 变中发挥重要作用 ${ }^{[3,4]}$, 已成为 HBV目前研究的焦点. 因此, 本研究选择HBx为靶点, 探讨其对TRAIL诱导 调亡的影响并初步探讨其分子机制，为进一步阐明 HBV相关疾病发病机制提供依据.

研究采用了两种实验方案来求证 HBx 对 TRAIL 诱导调亡的影响. 其一, 观察转染 $\mathrm{HBx}$ 真核表达载体 对 TRAIL 诱导 BEL7402 细胞调亡的影响. 台盼蓝染 色计数法显示 TRAIL 蛋白对 BEL7402, BEL7402-
cDNA3, BEL7402-HBx细胞均有剂量依赖性的细胞 毒效应，但在相同作用浓度下，TRAIL对BEL7402$\mathrm{HBx}$ 细胞较对照组细胞有更高的杀伤率. TUNEL结果 显示，在 $10 \mu \mathrm{LTRAIL}$ 蛋白作用下，BEL7402-HBx细 胞凋亡率可达 $(41.4 \% \pm 7.2) \%$, 显著高于BEL7402 细胞 $(13.8 \% \pm 6.5) \%$ 和BEL7402-cDNA3 细胞 $(14.2 \pm 4.7) \%$. 同时Caspase3 活性检测结果证明，经TRAIL作用之后 BEL7402-HBx 较对照组细胞有更高的Caspase3 活化 水平。其二，观察了硫代反义寡核苷酸 PS-asODNs/HBx对TRAIL诱导Hep G2.2.15 细胞调亡 的影响, TUNEL结果显示, PS-asODNs/HBx 与TRAIL 联合作用组Hep G2.2.15 细胞调亡率为 $(18.5 \% \pm 9.5) \%$, 显著低于 PS-rODNs 与 TRAIL 联合作用组 $(62.1 \% \pm 12) \%$. 以上结果显示, HBx基因的表达可增 强TRAIL诱导BEL7402 细胞的调亡反应, 而封闭 HBx 的表达可下调Hep G2.2.15 细胞的调亡率，二者在本 质上相一致并互为补充. 已有研究表明, $\mathrm{HBx}$ 蛋白除 具有反式激活的特性外, 还参与调节肝细胞的调亡. $\mathrm{Su}$ 等人 ${ }^{[6]}$ 发现低水平表达的 $\mathrm{HBx}$ 蛋白即可提高Chang, Hep G2 细胞对低剂量 TNF- $\alpha$ 诱导调亡的敏感性. FasL/Fas刺激所导致HBx 转基因鼠肝细胞的调亡率及 强度均显著强于同系鼠. 此外, HBx尚可逆转 $\mathrm{hBcl}-2$ 转基因鼠对Fas诱导调亡的敏感性 ${ }^{[9]}$. 因此, 在这个意 义上, HBx对TNF- $\alpha$, FasL 和TRAIL诱导的细胞调亡 有类似的作用.

但是, HBx 是通过何种方式影响TRAIL诱导的细 胞调亡呢? 众所周知, 机体存在 5 种TRAIL特异性结 合受体，其中包括两种死亡受体DR4/TRAIL-R1， DR5/TRAIL-R2, 介导TRAIL诱导的细胞调亡反应; 两种诱骗受体DcR1/TRAIL-R3，DcR2/TRAIL-R4，因 缺乏胞浆死亡结构域而不能向细胞内传递调亡信号; 一种可溶性受体OPG，被分泌在细胞外，负向调控 TRAIL诱导的细胞调亡反应 ${ }^{[10 \sim 12]}$. 研究表明, 细胞表

表 2 PS-asODNs/HBx 对HBV DNA复制的影响 ${ }^{a)}$

\begin{tabular}{|c|c|c|c|c|c|c|}
\hline & \multicolumn{2}{|c|}{ Hep G2.2.15 对照组 } & \multicolumn{2}{|c|}{ PS-rODNs 作用组 } & \multicolumn{2}{|c|}{ PS-asODNs/HBx 作用组 } \\
\hline & $24 \mathrm{~h}$ & $48 \mathrm{~h}$ & $24 \mathrm{~h}$ & $48 \mathrm{~h}$ & $24 \mathrm{~h}$ & $48 \mathrm{~h}$ \\
\hline $\begin{array}{l}\mathrm{HBV} \text { DNA }\left(\times 10^{4}\right. \\
\left.\text { copies } \cdot \mathrm{mL}^{-1}\right)\end{array}$ & $5.2 \pm 0.9$ & $19.8 \pm 4.2$ & $4.8 \pm 0.6$ & $17.6 \pm 3.3$ & $3.6 \pm 0.3$ & $4.5 \pm 0.7$ \\
\hline 抑制率 $/ \%$ & - & - & $7.7 \pm 1.3$ & $11.1 \pm 2.8^{*}$ & $30.2 \pm 1.1$ & $77.1 \pm 3.4^{* *}$ \\
\hline
\end{tabular}

a) 结果以均数土标准差表示. *示与 PS-rODNs作用组相比, $P<0.01 ; * *$ 示与PS-rODNs作用组相比, $P<0.001$ 
41

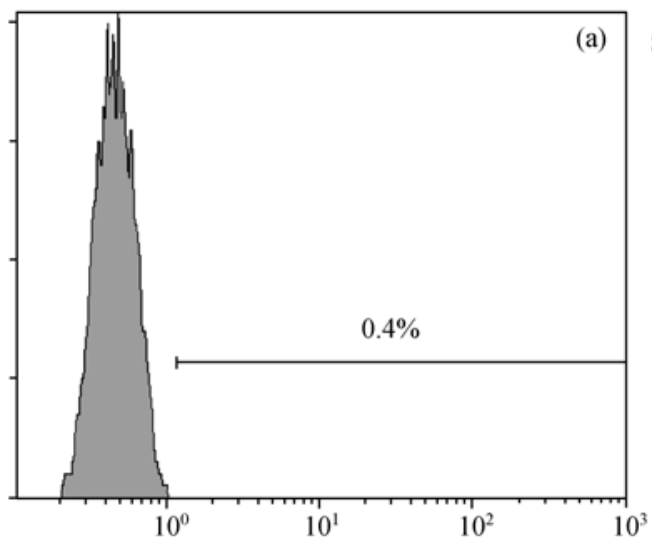

45

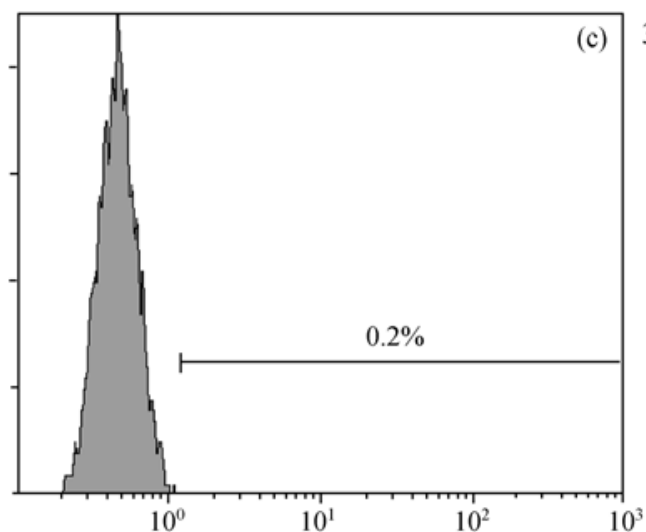

8

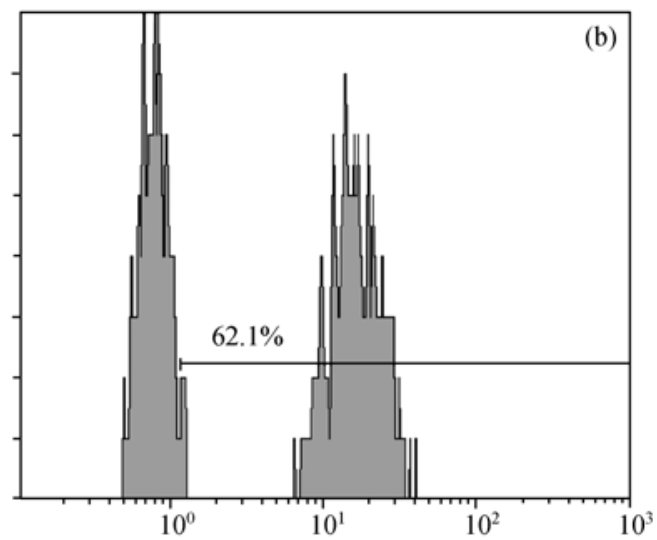

31

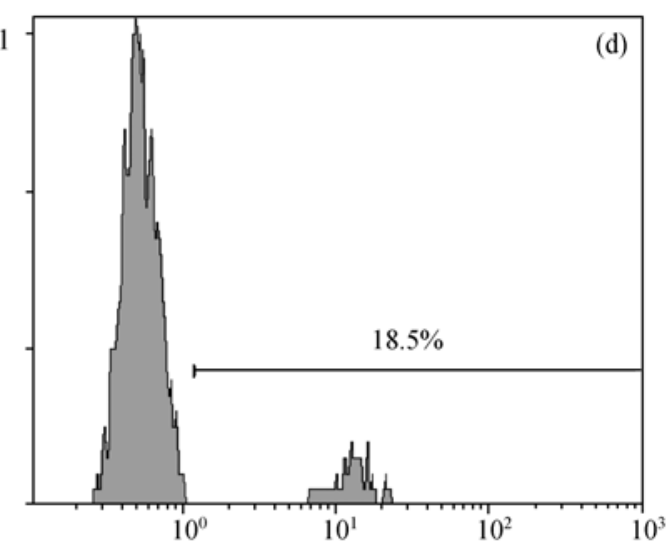

图 8 PS-asODNs/HBx 下调 HepG2.2.15 对 TRAIL 诱导调亡的敏感性 (a) $10 \mu \mathrm{mol} / \mathrm{L}$ of PS-rODNs 作用组; (b) $10 \mu \mathrm{mol} / \mathrm{L}$ of PS-rODNs 与 $100 \mu \mathrm{g} / \mathrm{L}$ of TRAIL 作用组; (c) PS-asODNs/HBx 作用组; (d) PS-asODNs $/ \mathrm{HBx}$ 与 $100 \mu \mathrm{g} / \mathrm{L}$ of TRAIL 作用组; *: 与(b)组相比, $P<0.05$;

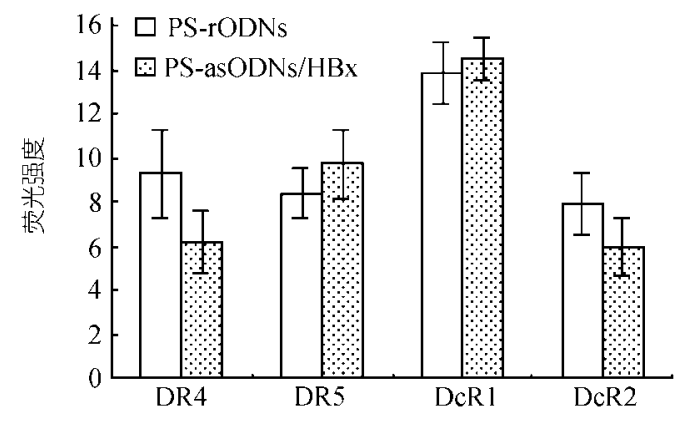

图 9 PS-asODNs/HBx 对 Hep G2.2.15 细胞 TRAIL 受体表达的影响

面死亡受体和诱骗受体的表达比例参与调节TRAIL 诱导细胞调亡的敏感性 ${ }^{[13]}$. 因此, 本研究利用流式细 胞术比较了BEL7402, BEL7402-cDNA3, BEL7402$\mathrm{HBx}$ 间和经PS-asODNs, PS-asODNs/HBx 作用的Hep
G2.2.15 细胞间表面TRAIL受体的表达. 结果表明, HBx基因的表达或者封闭不会影响TRAIL各受体的 表达，提示HBx对TRAIL诱导调亡敏感性的调节与其 受体表达无关. 尽管已有许多研究证实 $\mathrm{HBx}$ 具有诱导 细胞调亡的特性，但是其中的分子机制尚不明确. Shirakata等人 ${ }^{[14,15]}$ 研究发现, HBx表达后主要定位于 细胞线粒体, 引起线粒体膜电位的丢失, 最终导致细 胞色素 $\mathrm{C}$ 的释放和Caspase酶原的激活. 已有研究表 明，线粒体途径在TRAIL诱导的细胞调亡反应中发 挥重要作用, 因此, $\mathrm{HBx}$ 可能通过线粒体途径来影响 TRAIL诱导的细胞调亡. 此外, Kim等人 ${ }^{[16]}$ 发现 HBx 可与胞浆调亡抑制蛋白c-FLIP蛋白形成复合物，阻断 其抑制调亡的活性，导致细胞对低浓度TNF- $\alpha$ 的高敏 感性.已知 c-F L I P 蛋白的表达可抑制 F a s L, 
TNF和TRAIL所诱导的细胞调亡 ${ }^{[17]}$, 而且被认为是在 肝细胞肝癌中调节细胞生存与死亡的关键分子 ${ }^{[18]}$, 因此, c-FLIP蛋白亦可能为 HBx 影响TRAIL诱导调亡 的效应分子之一. 然而其详尽的分子机制尚值得进 一步研究.

本研究结果表明, HBx 蛋白的表达参与调节细胞 对 TRAIL 诱导调亡的敏感性, 但这种效应与 TRAIL 受体的表达无关，具体机制尚值得进一步探讨. 本研 究将为进一步阐明 HBV 感染相关疾病发病机制提供 有价值的参考.

\section{参考 文献}

1 Wiley S R, Schooley K, Smolask P J, et al. Identification and characterization of new member of the TNF family that induces apoptosis. Immunity, 1995, 3(6): 673 682[DOI]

2 Gruss H J. Molecular, structural, and biological characteristics of the tumor necrosis factor ligand superfamily. Int J Clin Lab Res, 1996, 26(3): 143 159

3 Arbuthnot P, Kew M. Hepatitis B virus and hepatocellular carcinoma. Int J Exp Pathol, 2001, 82(2): 77 100

4 Murakami S. Hepatitis B virus X protein: a multifunctional viral regulator. J Gastroenterol, 2001, 36(10): 651 660[DOI]

5 Tanaka Y, Kanai F, Kawakami T, et al. Interaction of the hepatitis $\mathrm{B}$ virus $\mathrm{X}$ protein $(\mathrm{HBx})$ with heat shock protein 60 enhances HBx-mediated apoptosis. Biochem Biophys Res Commun, 2004, 318(2): 461 469[DOI]

6 Su F, Schneider R J. Hepatitis B virus HBx protein sensitizes cells to apoptotic killing by tumor necrosis factor $\alpha$. Proc Natl Acad Sci USA, 1997, 94(16): 8744 8749[DOI]

7 Galle P R, Hofmann W J, Walczak H, et al. Involvement of the CD95(APO-1/Fas) receptor and ligand in liver damage. J Exp Med, 1995, 182(5): 1223 1230[DOI]
8 Kagi D, Vignaus F, Ledermann B, et al. Fas and perforin pathways as major mechanisms of T cell-mediated cytotoxicity. Science, 1994, 265(5171): 528 530

9 Terradillos O, Coste A L, Pollicino T, et al. The hepatitis B virus $\mathrm{X}$ protein abrogates $\mathrm{Bcl}-2$-mediated protection against Fas apoptosis in the liver. Oncogene, 2002, 21(3): 377 386[DOI]

10 Pan G, O'Rourke K, Chinnaiyan A M, et al. The receptor for the cytotoxic ligand TRAIL. Science, 1997, 276(5309): 111 113[DOI]

11 Sheridan J P, Marsters S A, Pitti R M, et al. Control of TRAILinduced apoptosis by a family of signaling and decoy receptors. Science, 1997, 277(5327): 818 821[DOI]

12 Emery J G, McDonnell P, Burke M B, et al. Osteoprotegrin is a receptor for the cytotoxic ligand TRAIL. J Biol Chem, 1998, 273(23): 14363 14367[DOI]

13 Degli-Esposti M. To die or not to die - the quest of the TRAIL receptors. J Leukoc Biol, 1999, 65(5): 535 542

14 Shirakata Y, Kioke K. Hepatitis B virus X protein induces cell death by causing loss of mitochondrial membrane potential. J Biol Chem, 2003, 278(24): 23071 23078

15 Takada S, Shirakata Y, Kaneniwa N, et al. Associated of hepatitis $\mathrm{B}$ virus $\mathrm{X}$ protein with mitochondria causes mitochondria aggregation at the nuclear periphery, leading to cell death. Oncogene, 1999, 18: 6965 6973[DOI]

16 Kim KH, Seong B. Pro-apoptotic function of HBV X protein is mediated by interaction with c-FLIP and enhancement of deathinducing signal. EMBO J, 2003, 22(9): 1 13[DOI]

17 Thome M, Schneider P, Hofmann K, et al. Viral FLICE-inhibitory proteins(FLIPs) prevent apoptosis induced by death receptors. Nature, 1997, 386(6624): 517 521[DOI]

18 Okano H, Shiraki K, Inoue H, et al. Cellular FLICE/caspase8 -inhibitory protein as a principal regulator of cell death and survival in human hepatocellular carcinoma. Lab Invest, 2003, 83(7): 1033 1043[DOI] 